\title{
A continuum theory for first-order phase transitions based on the balance of structure order
}

\author{
M. Fabrizio ${ }^{1}$, C. Giorgi ${ }^{2}$ and A. Morro ${ }^{3, *, \dagger}$ \\ ${ }^{1}$ Department of Mathematics, Piazza di Porta S. Donato, 5, 40126 Bologna, Italy \\ ${ }^{2}$ Department of Mathematics, Via Valotti 9, 25133 Brescia, Italy \\ ${ }^{3}$ University of Genova, DIBE, Via Opera Pia, 11a, 16145 Genova, Italy
}

Communicated by B. Straughan

\section{SUMMARY}

First-order phase transitions are modelled by a non-homogeneous, time-dependent scalar-valued order parameter or phase field. The time dependence of the order parameter is viewed as arising from a balance law of the structure order. The gross motion is disregarded and hence the body is regarded merely as a heat conductor. Compatibility of the constitutive functions with thermodynamics is exploited by expressing the second law through the classical Clausius-Duhem inequality. First, a model for conductors without memory is set up and the order parameter is shown to satisfy a maximum theorem. Next, heat conductors with memory are considered. Different evolution problems are established through a system of differential equations whose form is related to the manner in which the memory property is represented. Copyright (c) 2007 John Wiley \& Sons, Ltd.

KEY WORDS: phase changes; evolution equation; structure order; heat conductor

\section{INTRODUCTION}

In any phase transition, the states of the two phases are endowed with different structures or symmetries. Indeed, for a number of materials, the structure order below a critical temperature is greater than above the temperature. In the solid-fluid transition, the solid phase has a greater structure order due to the crystal symmetry group. In ferromagnetism below the critical temperature, the magnetic moments are aligned, and in superconductivity and superfluidity, the order is associated with the structure of the velocity. Following Landau and Ginzburg [1], we characterize the structure order through an order parameter which is a scalar quantity $\varphi \in[0,1]$.

\footnotetext{
*Correspondence to: A. Morro, University of Genova, DIBE, Via Opera Pia, 11a, 16145 Genova, Italy.

${ }^{\dagger}$ E-mail: morro@dibe.unige.it
} 
In order to establish ideas, we let $\varphi$ increase with the structure order so that $\varphi=0$ in the less-ordered phase. Often $\varphi$ is identified with the concentration. Hence, for example, in the ice-water transition, $\varphi$ is the ice concentration and $\varphi=0$ and 1 in the fluid and solid phases, respectively.

The aim of this paper is to model the evolution of $\varphi$ as a non-equilibrium phase transition induced by temperature or pressure variations. The evolution is allowed to be non-homogeneous and hence the order parameter $\varphi$ is a time-dependent field, currently named the phase field.

In this paper, we follow the view that the evolution of $\varphi$ is a balance law for the structure order. This view may appear reminiscent of those of Fried and Gurtin [2,3] and Frémond et al. (see, e.g. $[4,5]$ ) in which the phase transition is based on a balance equation of microforces. The difference mainly lies in our view of the structure order as a macroscopic and observable quantity that enters a balance equation. Upon appropriate characterizations, the balance law eventually results in the pertinent Ginzburg-Landau equation.

The approaches to the modelling of phase transitions can be characterized through the corresponding framework for compatibility with thermodynamics. In [6], the phase field is viewed as an internal variable and an entropy extra-flux is allowed to enter the second law. The model so established is shown to involve the free energy in the rescaled form (see [7]). In [2,3], the crucial step is the introduction of microforces and the associated power in the balance of energy, whereas the second law is kept in the classical Clauius-Duhem form. In [5], the modelling equations are established through the principle of virtual power involving the microscopic velocities and the virtual power of acceleration.

In essence, the present approach is based on a further balance law that of the structure order. Such an equation may also be viewed as the evolution equation for the phase field and involves a vector structure flux. Such a vector field is associated with an appropriate power in the energy balance. Compatibility with thermodynamics is proved by expressing the second law in the classical Clausius-Duhem inequality. Preliminarily, we set up a model for materials without memory; next, we insert memory properties for the dependence on the temperature gradient. It is a remarkable result that the evolution equation, for the order parameter, allows us to establish a maximum theorem.

Notation: A superposed dot and $\partial_{t}$ stand for time differentiation. Also, $\nabla$ is the gradient operator, $\nabla$. is the divergence and $\Delta$ is the Laplacian. A prime denotes the derivative with respect to the pertinent variable.

\section{BALANCE LAW FOR THE STRUCTURE ORDER}

We consider a body $\mathscr{B}$. The formulation of the balance equations for a material with a structure order, undergoing phase transitions, is established by analogy with the standard balance laws, in continuum mechanics, which may be viewed as the equality among internal and external actions for any part of a body.

Following the general scheme of continua, for any part $S \subset \mathscr{B}$ we let the external structure-order action $\mathscr{A}^{\mathrm{e}}(S)$, on the material system in $S$, consist of a volume part and a surface part, namely

$$
\mathscr{A}^{\mathrm{e}}(S)=\int_{\partial S} \mathbf{p} \cdot \mathbf{n} \mathrm{d} a+\int_{S} \rho \sigma \mathrm{d} v
$$


where $\mathbf{p}$ is the structure-order flux and $\sigma$ is the structure-order supply. Let $\mathscr{A}^{\mathrm{i}}(S)$ be the internal structure-order action with density $\kappa$ per unit mass:

$$
\mathscr{A}^{\mathrm{i}}(S)=\int_{S} \rho \kappa \mathrm{d} v
$$

The balance of structure order is expressed by the equality of the two actions whence, for any $S \subset \mathscr{B}$,

$$
\int_{S} \rho \kappa \mathrm{d} v=\int_{\partial S} \mathbf{p} \cdot \mathbf{n} \mathrm{d} a+\int_{S} \rho \sigma \mathrm{d} v
$$

The divergence theorem and the arbitrariness of $S$ give the local form

$$
\rho \kappa=\nabla \cdot \mathbf{p}+\rho \sigma
$$

Multiplying (2) by $\dot{\varphi}$, we obtain

$$
\rho \kappa \dot{\varphi}+\mathbf{p} \cdot \nabla \dot{\varphi}=\nabla \cdot(\mathbf{p} \dot{\varphi})+\rho \sigma \dot{\varphi}
$$

This suggests that we consider the left-hand side of (3) as the internal power (density) and the right-hand side as the external power:

$$
\mathscr{P}^{\mathrm{i}}=\rho \kappa \dot{\varphi}+\mathbf{p} \cdot \nabla \dot{\varphi}, \quad \mathscr{P}^{\mathrm{e}}=\nabla \cdot(\mathbf{p} \dot{\varphi})+\rho \sigma \dot{\varphi}
$$

The density $\kappa$ of internal action is allowed to depend on $\varphi$ and $\dot{\varphi}$ in the form

$$
\rho \kappa=\gamma \dot{\varphi}+f(\varphi)+u g(\varphi)
$$

where $\gamma$ is a positive constant, $f$ and $g$ are functions so far unrestricted and $u$ is a parameter controling the transition.

For definiteness, the parameter $u$ may represent the temperature and the pressure in solid-fluid and fluid-vapour transitions, the temperature and the magnetic field in conducting-superconducting transitions, and, possibly, the square of the velocity in turbulence.

Since the structure flux $\mathbf{p}$ arises because of the inhomogeneity of $\varphi$, it is natural to let $\mathbf{p}$, be given by the constitutive equation:

$$
\mathbf{p}=v \nabla \varphi
$$

where $v$ is a positive constant. Also, for simplicity we let $\sigma=0$.

Owing to (2), (5) and (6), we obtain

$$
\gamma \dot{\varphi}+f(\varphi)+u g(\varphi)=v \Delta \varphi
$$

which may be viewed as the Ginzburg-Landau equation for the transition. Since we disregard the gross motion, henceforth we look only at phase transitions induced by the temperature. It is then natural to identify $u$ with the ratio of the absolute temperature $\theta$ over the (absolute) transition temperature $\theta_{c}$. Hence, we write

$$
\gamma \dot{\varphi}+f(\varphi)+\frac{\theta}{\theta_{c}} g(\varphi)=v \Delta \varphi
$$

Upon the choice (5), Equation (2) may be viewed as the evolution equation for $\varphi$. We now need a further differential equation involving $\theta$ so that, along with (7), a system of equations for $\varphi$ and $\theta$ is available. That is why we now examine the balance of energy. 


\section{BALANCE OF ENERGY}

Having in mind a thermally induced phase transition, we express the balance of energy by assuming that the external heat action $\mathscr{H}^{\mathrm{e}}$ results from a heat flux vector $\mathbf{q}$ and a heat supply $r$ :

$$
\mathscr{H}^{\mathrm{e}}(S)=-\int_{\partial S} \mathbf{q} \cdot \mathbf{n} \mathrm{d} a+\int_{S} \rho r \mathrm{~d} v
$$

Denote by $h$ the specific internal heat action and

$$
\mathscr{H}^{\mathrm{i}}(S)=\int_{S} \rho h \mathrm{~d} v
$$

Equality of internal and external actions

$$
\int_{S} \rho h \mathrm{~d} v=-\int_{\partial S} \mathbf{q} \cdot \mathbf{n} \mathrm{d} a+\int_{S} \rho r \mathrm{~d} v
$$

for any sub-body $S$ results in

$$
\rho h=-\nabla \cdot \mathbf{q}+\rho r
$$

The heat flux $\mathbf{q}$ is denoted by the form

$$
\mathbf{q}=-k(\theta) \nabla \theta
$$

where $k$ is a temperature-dependent conductivity. In the classical Fourier law for heat conductors $k$ is a constant, whereas for insulators, and hence for water [8], $k$ is often assumed to be linear, $k(\theta)=k_{0} \theta$.

The internal structure-order density $\mathscr{P}^{\mathrm{i}}$ is viewed as an internal action power and then, by the first law of thermodynamics, we write

$$
\rho \dot{e}=\rho h+\mathscr{P}^{\mathrm{i}}
$$

It then follows from (9) that

$$
\rho(\dot{e}-\kappa \dot{\varphi})-\mathbf{p} \cdot \nabla \dot{\varphi}=k^{\prime}|\nabla \theta|^{2}+k \Delta \theta+\rho r
$$

the prime denoting the derivative.

Let $F$ and $G$ be the integrals of $f$ and $g$. Upon substitution for $\kappa$ and $\mathbf{p}$, we obtain

$$
\rho \dot{e}-\dot{F}-\frac{\theta}{\theta_{c}} \dot{G}-\gamma \dot{\varphi}^{2}-\frac{1}{2} \nu \partial_{t}|\nabla \varphi|^{2}=k^{\prime}|\nabla \theta|^{2}+k \Delta \theta+\rho r
$$

For convenience we let

$$
F(0)=0, \quad G(0)=0
$$

Also, henceforth we will regard $\rho$ as a constant and hence we let $\rho=1$. 


\section{THERMODYNAMIC RESTRICTIONS}

Let $\eta$ be the entropy density. The second law of thermodynamics is taken in the form of the Clausius-Duhem inequality

$$
\dot{\eta} \geqslant-\nabla \cdot \frac{\mathbf{q}}{\theta}+\frac{\rho r}{\theta}
$$

to hold for all functions compatible with (7) and (11).

By replacing $-\nabla \cdot \mathbf{q}+r$ with $\dot{e}-\mathscr{P}^{\mathrm{i}}$ (see (9)) and using the free energy $\psi=e-\theta \eta$, we have

$$
\dot{\psi}+\eta \dot{\theta}-\kappa \dot{\varphi}-\mathbf{p} \cdot \nabla \dot{\varphi}+\frac{1}{\theta} \mathbf{q} \cdot \nabla \theta \leqslant 0
$$

The free energy $\psi$ and the entropy $\eta$ are assumed to depend on $\theta, \varphi, \nabla \varphi$. Hence upon substitution of $\mathscr{P}^{i}$, we obtain

$$
\left(\psi_{\theta}+\eta\right) \dot{\theta}-\gamma \dot{\varphi}^{2}+\left(\psi_{\varphi}-f-\frac{\theta}{\theta_{c}} g\right) \dot{\varphi}+\left(\psi_{\nabla \varphi}-v \nabla \varphi\right) \cdot \nabla \dot{\varphi}-\frac{1}{\theta} k|\nabla \theta|^{2} \leqslant 0
$$

We now evaluate the restrictions placed by the second law through (13). The arbitrariness of $\dot{\theta}$ and the linearity in $\dot{\theta}$ imply that

$$
\eta=-\psi_{\theta}
$$

Now, $\dot{\varphi}$ is subject to (7) and hence may be chosen arbitrarily because $\Delta \varphi$ does so. The arbitrariness of $\nabla \dot{\varphi}$ implies that

$$
\psi_{\nabla \varphi}=v \nabla \varphi
$$

Now, letting $\nabla \theta=0$ we have

$$
-\gamma \dot{\varphi}^{2}+\left(\psi_{\varphi}-f-\frac{\theta}{\theta_{c}} g\right) \dot{\varphi} \leqslant 0
$$

If $\psi_{\varphi}-f-g \theta / \theta_{c} \neq 0$, then by letting $|\dot{\varphi}|$ be sufficiently small, we conclude that the linear term predominates and hence the inequality is contradicted. Hence, it follows that

$$
f+\frac{\theta}{\theta_{c}} g=\psi_{\varphi}
$$

The reduced inequality

$$
-\theta \gamma \dot{\varphi}^{2}-k|\nabla \theta|^{2} \leqslant 0
$$

holds if and only if

$$
\gamma \geqslant 0, \quad k \geqslant 0
$$

Conversely, it is obvious that the restrictions (14)-(17) are sufficient for the validity of the ClausiusDuhem inequality (13), subject to (7) and (11). 


\section{Remark}

In other approaches (see [6]), the phase field $\varphi$ is found to satisfy the differential equation:

$$
\dot{\varphi}=-\frac{1}{\gamma}\left[\frac{\psi_{\varphi}}{\theta}-\nabla \cdot\left(\frac{1}{\theta} \psi_{\nabla \varphi}\right)\right]
$$

In such a case, the evolution equation (7) follows by letting $\gamma$ be a constant and

$$
\psi=\theta\left[H(\theta)+F(\varphi)+\frac{\theta}{\theta_{c}} G(\varphi)+\frac{1}{2} v|\nabla \varphi|^{2}\right]
$$

\section{SOLID-FLUID TRANSITION}

For the sake of definiteness, we have to choose a form of the free energy function $\psi$. Let

$$
B(\theta)=c \theta(\ln \theta-1)
$$

Having in mind the description of the ice-water transition, we mention that the Penrose-Fife model [9] is based on the free energy:

$$
\psi_{\mathrm{s}}(\theta)=L \frac{\theta-\theta_{c}}{\theta_{c}}-B(\theta)
$$

for the solid phase and the free energy

$$
\psi_{1}(\theta)=-B(\theta)
$$

for the liquid phase. This model is corroborated by the feature that the corresponding internal energies $e_{\mathrm{s}}, e_{1}$ satisfy

$$
e_{1}=e_{\mathrm{S}}+L, \quad e_{1}=-B(\theta)+\theta B^{\prime}(\theta)
$$

$L$ playing the role of latent heat, consistent with the description of first-order transitions. As $\varphi \in[0,1]$, the free energy is taken as

$$
\psi(\theta, \varphi)=L\left[h(\varphi) \frac{\theta-\theta_{c}}{\theta_{c}}+w(\varphi)\right]-B(\theta)
$$

where $h(0)=0, h(1)=1$ and $w(0)=w(1)=0$.

Here, we set up a more detailed model by generalizing the dependence on $\theta$ and $\varphi$. In view of non-uniform configurations, we define $\psi$ in the form:

$$
\psi(\theta, \varphi, \nabla \varphi)=-B(\theta)+F(\varphi)+\frac{\theta}{\theta_{c}} G(\varphi)+\frac{1}{2} v|\nabla \varphi|^{2}
$$

whence the Penrose-Fife model follows by letting $G(\varphi)=L h(\varphi), F(\varphi)=L w(\varphi)-G(\varphi)$ and $v=0$. By (14), we have

$$
\eta(\theta, \varphi)=B^{\prime}(\theta)-\frac{1}{\theta_{c}} G(\varphi)
$$


The ice-water transition suggests that we choose (see $[6,10])$

$$
F(\varphi)=12 L\left(\frac{1}{4} \varphi^{4}-\frac{1}{3} \varphi^{3}\right), \quad G(\varphi)=12 L\left(\frac{1}{4} \varphi^{4}-\frac{2}{3} \varphi^{3}+\frac{1}{2} \varphi^{2}\right)
$$

so that

$$
W(\varphi):=F(\varphi)+G(\varphi)=6 L \varphi^{2}(1-\varphi)^{2}
$$

is the double-well potential with $3 L / 8$ as the maximum value, at $\varphi=\frac{1}{2}$. Hence, we can write the free energy in the form:

$$
\psi(\theta, \varphi, \nabla \varphi)=-B(\theta)+W(\varphi)+\frac{\theta-\theta_{c}}{\theta_{c}} G(\varphi)+\frac{1}{2} v|\nabla \varphi|^{2}
$$

By (14), we have

$$
\eta=B^{\prime}(\theta)-\frac{1}{\theta_{c}} G(\varphi)
$$

and hence, because

$$
\begin{gathered}
-B(\theta)+\theta B^{\prime}(\theta)=c \theta \\
e=c \theta+\theta B^{\prime}(\theta)+F(\varphi)+\frac{1}{2} v|\nabla \varphi|^{2}
\end{gathered}
$$

\subsection{Latent heat}

It is customary to define the latent heat as the minimum energy released or absorbed during a homogeneous and quasi-static change of state. The phase transition occurs at the transition temperature $\theta_{c}$. Let $L$ be the specific latent heat, per unit mass, and let the phase transition, $\varphi$ from 1 to 0 , occur in the time interval $\left[t_{1}, t_{2}\right], \varphi\left(t_{1}\right)=1, \varphi\left(t_{2}\right)=0$. In a uniform configuration, by (19) and (11) we have

$$
-\dot{G}-\gamma \dot{\varphi}^{2}=r
$$

Integration over $\left[t_{1}, t_{2}\right]$ gives

$$
\int_{t_{1}}^{t_{2}} r \mathrm{~d} t=-G(0)+G(1)-\gamma \int_{t_{1}}^{t_{2}} \dot{\varphi}^{2} \mathrm{~d} t
$$

For quasi-static processes, the integral of $\dot{\varphi}^{2}$ is as small as we please and, accordingly, we have

$$
L=G(1)-G(0)
$$

This result is consistent with the definition of $L$ as

$$
L=\theta_{c}\left[\eta\left(\theta_{c}, 0\right)-\eta\left(\theta_{c}, 1\right)\right]
$$

\subsection{Thermal potential}

To select the function $C(\theta)=-B(\theta)+\theta B^{\prime}(\theta)$, we observe that $c(\theta)=C^{\prime}(\theta)$ is the specific heat. Now, according to the Debye theory the specific heat is a function of the ratio $\theta / \theta_{\mathrm{D}}, \theta_{\mathrm{D}}$ being the 
Debye temperature, namely

$$
c(\theta)=\bar{c} D\left(\theta / \theta_{\mathrm{D}}\right), \quad \bar{c}>0
$$

the function $D$ being defined by

$$
D(\xi)=\xi^{3} \int_{0}^{1 / \xi} \frac{x^{3}}{\exp (x)-1} \mathrm{~d} x-\frac{1}{4 \xi[\exp (1 / \xi)-1]}
$$

As $\theta$ is much smaller than $\theta_{\mathrm{D}}$, we may use the approximation

$$
c(\theta)=c_{0} \theta^{3}, \quad c_{0}>0
$$

Correspondingly $e, \psi$ and $\eta$ take the form

$$
\begin{aligned}
e(\theta, \varphi, \nabla \varphi) & =\frac{1}{4} c_{0} \theta^{4}+F(\varphi)+\frac{1}{2} v|\nabla \varphi|^{2} \\
\psi(\theta, \varphi, \nabla \varphi) & =-\frac{1}{12} c_{0} \theta^{4}+F(\varphi)+\frac{\theta}{\theta_{c}} G(\varphi)+\frac{1}{2} v|\nabla \varphi|^{2} \\
\eta(\theta, \varphi) & =\frac{1}{3} c_{0} \theta^{3}-\frac{1}{\theta_{c}} G(\varphi)
\end{aligned}
$$

At very low temperatures, the electronic contribution to the specific heat is significant and results in a linear term so that

$$
c(\theta)=c_{0} \theta^{3}+c_{1} \theta, \quad c_{0}, c_{1}>0
$$

When the linear term becomes predominant, the internal energy and the free energy are quadratic in $\theta$, whereas the entropy is linear.

In the limit of high temperatures, $\theta \gg \theta_{\mathrm{D}}$, the specific heat becomes a constant (Dulong-Petit law) and

$$
c(\theta)=\hat{c}>0, \quad \hat{c}=\frac{1}{3} \bar{c}
$$

In such a case

$$
\begin{aligned}
e(\theta, \varphi, \nabla \varphi) & =\hat{c} \theta+F(\varphi)+\frac{1}{2} v|\nabla \varphi|^{2} \\
\psi(\theta, \varphi, \nabla \varphi) & =-\hat{c} \theta \ln \theta+F(\varphi)+\frac{\theta}{\theta_{c}} G(\varphi)+\frac{1}{2} v|\nabla \varphi|^{2} \\
\eta(\theta, \varphi) & =\hat{c}(1+\ln \theta)-\frac{1}{\theta} G(\varphi)
\end{aligned}
$$

\section{EVOLUTION PROBLEM}

We now investigate the evolution problem associated with the phase transition. To this end, we observe that the balance of energy in the form (11) and the choice (20) of $e$ give

$$
\hat{c} \dot{\theta}-\frac{\theta}{\theta_{c}} g \dot{\varphi}-\gamma \dot{\varphi}^{2}-k^{\prime}|\nabla \theta|^{2}-k \theta \Delta \theta-r=0
$$


Also, by (18) we have $f(\varphi)=\varphi^{2}(1-\varphi), g(\varphi)=\varphi(\varphi-1)^{2}$. Hence, we can write $(8)$ as

$$
\gamma \dot{\varphi}+\varphi(\varphi-1)\left(\varphi+(\varphi-1) \frac{\theta}{\theta_{c}}\right)-v \Delta \varphi=0
$$

Equations (21) and (22) constitute the system of evolution equations for the variables $\theta, \varphi$.

The evolution problem for $\theta$ and $\varphi$ in the domain $\Omega \times[0, T]$ consists of the differential equations (21), (22) and the boundary conditions

$$
\theta_{\partial \Omega_{1}}=\hat{\theta},\left.\quad \nabla \theta \cdot \mathbf{n}\right|_{\partial \Omega_{2}}=0,\left.\quad \nabla \varphi \cdot \mathbf{n}\right|_{\partial \Omega}=0
$$

where $\partial \boldsymbol{\Omega}_{1}$ and $\partial \boldsymbol{\Omega}_{2}$ are a partition of $\partial \boldsymbol{\Omega}$ such that $\overline{\partial \boldsymbol{\Omega}_{1} \cup \partial \boldsymbol{\Omega}_{2}}=\partial \boldsymbol{\Omega}$ and $\partial \boldsymbol{\Omega}_{1} \cap \partial \boldsymbol{\Omega}_{2}=\emptyset$.

\section{Theorem 1}

Every solution $(\varphi, \theta), \theta>0$, to the evolution problem, subject to the initial condition $\varphi(\mathbf{x}, 0)=$ $\varphi_{0}(\mathbf{x}) \in[0,1]$, satisfies $\varphi(\mathbf{x}, t) \in[0,1]$ almost everywhere in $\Omega \times[0, T]$.

Proof

First we show that $\varphi \geqslant 0$ and next that $\varphi \leqslant 1$. Let

$$
\varphi_{-}:=\max (-\varphi, 0)
$$

Multiply (22) by $\varphi_{-}$to obtain

$$
\gamma \varphi_{-} \dot{\varphi}=v \nabla \cdot\left(\varphi_{-} \nabla \varphi\right)-v \nabla \varphi_{-} \cdot \nabla \varphi+\varphi_{-} \varphi(1-\varphi)\left(\varphi+(\varphi-1) \theta / \theta_{c}\right)
$$

Now,

$$
\varphi_{-} \varphi(1-\varphi)(\varphi-1) \theta / \theta_{c}= \begin{cases}0, & \varphi>0 \\ \varphi^{2}(1-\varphi)^{2} \theta / \theta_{c}, & \varphi<0\end{cases}
$$

Hence,

$$
\gamma \varphi_{-} \dot{\varphi} \geqslant v \nabla \cdot\left(\varphi_{-} \nabla \varphi\right)-v \nabla \varphi_{-} \cdot \nabla \varphi+\varphi_{-} \varphi^{2}(1-\varphi)
$$

Since

$$
\dot{\varphi}_{-}= \begin{cases}0, & \varphi>0 \\ -\dot{\varphi}, & \varphi<0\end{cases}
$$

then

$$
\varphi_{-} \dot{\varphi}_{-}=-\varphi_{-} \dot{\varphi}
$$

Likewise

$$
\nabla \varphi_{-} \cdot \nabla \varphi_{-}=-\nabla \varphi_{-} \cdot \nabla \varphi
$$

Consequently, integration of (25) over $\Omega$ and the boundary conditions (23) yield

$$
\frac{1}{2} \int_{\Omega} \gamma\left(\varphi_{-}^{2}\right) \mathrm{d} v=-\int_{\Omega} v\left|\nabla \varphi_{-}\right|^{2} \mathrm{~d} v-\int_{\Omega} \varphi_{-} \varphi^{2}(1-\varphi) \mathrm{d} v
$$


For any $\mathbf{x} \in \Omega$, the initial condition $\varphi(\mathbf{x}, 0) \in[0,1]$ implies that

$$
\varphi_{-}(\mathbf{x}, 0)=0
$$

Integration in time over $[0, t]$ then provides

$$
\frac{1}{2} \int_{\Omega} \gamma \varphi_{-}^{2}(\mathbf{x}, t) \mathrm{d} v+\int_{0}^{t} \int_{\Omega}\left[v\left|\nabla \varphi_{-}\right|^{2}+\varphi_{-} \varphi^{2}(1-\varphi)\right](\mathbf{x}, \tau) \mathrm{d} v \mathrm{~d} \tau \leqslant 0
$$

By definition, we have

$$
\varphi_{-}(1-\varphi) \geqslant 0
$$

As a consequence, it follows that

$$
\int_{\Omega} \gamma \varphi_{-}^{2}(\mathbf{x}, t) \mathrm{d} v \leqslant 0
$$

whence

$$
\varphi_{-}(\mathbf{x}, t)=0 \quad \forall \mathbf{x} \in \Omega \quad \forall t>0
$$

and then $\varphi \geqslant 0 \forall t>0$ in $\Omega$. We now show that $\varphi \leqslant 1 \forall t>0$. To this end, multiply (22) by

$$
(\varphi-1)_{+}:=\max (\varphi-1,0)
$$

to obtain

$$
\gamma(\varphi-1)_{+} \dot{\varphi}=v(\varphi-1)_{+} \Delta \varphi+(\varphi-1)_{+} \varphi(1-\varphi)\left(\varphi+(\varphi-1) \theta / \theta_{c}\right)
$$

By paralleling the previous procedure, we have

$$
\frac{1}{2} \gamma \int_{\Omega}\left((\varphi-1)_{+}^{2}\right) \mathrm{d} v \leqslant-v \int_{\Omega}\left|\nabla(\varphi-1)_{+}\right|^{2} \mathrm{~d} v-\int_{\Omega} \varphi^{2}(\varphi-1)_{+}^{2} \mathrm{~d} v
$$

The initial condition $\varphi(\mathbf{x}, 0) \in[0,1]$ implies that

$$
(\varphi-1)_{+}(\mathbf{x}, 0)=0
$$

Consequently, integration in time over $[0, t]$ leads to

$$
(\varphi-1)_{+}(\mathbf{x}, t)=0 \quad \forall \mathbf{x} \in \Omega \quad \forall t>0
$$

and hence $\varphi \leqslant 1 \forall t>0$ in $\Omega$.

Based upon the approximation that $\gamma \dot{\varphi}^{2}$ and $k|\nabla \theta|^{2}$ are neglected in (21) and dividing by $\theta$, the balance of energy (21) reduces to

$$
\hat{c} \partial_{t} \ln \theta-\frac{1}{\theta_{c}} g \dot{\varphi}-k_{0} \Delta \theta-\frac{r}{\theta}=0
$$

By applying Theorem 1, well posedness of the resulting system of equations (22), (26), with the conditions (23), may be established (see [11]). 


\section{HEAT CONDUCTORS WITH MEMORY}

We now look at a model of phase transition in rigid heat conductors with memory. A Jeffreys-type conductor (see [12]) obeys the differential equation

$$
\dot{\mathbf{q}}+\varepsilon \mathbf{q}=-k_{0} \nabla \dot{\theta}-\varepsilon k_{1} \nabla \theta
$$

This equation provides a Fourier law both in the fast limit, i.e. $\varepsilon \rightarrow 0$, and in the slow limit, i.e. $\varepsilon \rightarrow \infty$

$$
\mathbf{q} \rightarrow-k_{0} \nabla \theta, \quad \mathbf{q} \rightarrow-k_{1} \nabla \theta
$$

Integration of $(27)$ over $(-\infty, t)$ yields

$$
\mathbf{q}(t)=-k_{0} \nabla \theta(t)-\int_{0}^{\infty} k_{\varepsilon}(s) \nabla \theta(t-s) \mathrm{d} s
$$

where

$$
k_{\varepsilon}(s)=\varepsilon\left(k_{0}-k_{1}\right) \exp (-\varepsilon s), \quad s \in \mathbb{R}^{+}
$$

By paralleling non-linear generalizations of the Maxwell-Cattaneo model (see [13,14]), we consider the vector variable

$$
\mathbf{v}=\alpha(\theta) \mathbf{q}
$$

and let $\mathbf{v}$ be given by the evolution equation:

$$
\dot{\mathbf{v}}+\varepsilon \mathbf{v}=-k_{0} \nabla \dot{\theta}-\varepsilon k_{1} \nabla \theta
$$

Upon integration over $(-\infty, t]$, we have

$$
\mathbf{q}(t)=-\frac{1}{\alpha(\theta(t))}\left[k_{0} \nabla \theta(t)+\int_{0}^{\infty} k_{\varepsilon}(s) \nabla \theta(t-s) \mathrm{d} s\right]
$$

The identifications

$$
k(\theta)=\frac{k_{0}}{\alpha(\theta)}, \quad h(s)=\frac{k_{\varepsilon}(s)}{k_{0}}
$$

allow $\mathbf{q}$ to be given by

$$
\mathbf{q}(t)=-k(\theta(t))\left[\nabla \theta(t)+\int_{0}^{\infty} h(s) \nabla \theta(t-s) \mathrm{d} s\right]
$$

The constitutive equation (28) shows that $\mathbf{q}$ depends on the actual value $\nabla \theta(t)$ and on the history of $\nabla \theta$ through the same conductivity coefficient $k(\theta)$. As a comment, the linear model by Coleman and Gurtin [15] is recovered by letting the conductivity $k$ be a constant.

We assume $h \in W^{2,1}\left(\mathbb{R}^{+}\right) \cap H^{1}\left(\mathbb{R}^{+}\right)$. Also, to simplify the notation, henceforth we let $\mathbf{g}=\nabla \theta$ and denote by $\mathbf{g}^{t}$ the history up to the time $t$, i.e.

$$
\mathbf{g}^{t}(\mathbf{x}, s):=\mathbf{g}(\mathbf{x}, t-s), \quad s \in \mathbb{R}^{+}
$$


For later developments, it is convenient to consider

$$
\tilde{\mathbf{g}}^{t}(s)=\int_{0}^{s} \mathbf{g}(t-\xi) \mathrm{d} \xi=\int_{t-s}^{t} \mathbf{g}(\tau) \mathrm{d} \tau
$$

we call $\tilde{\mathbf{g}}$ the summed history of $\mathbf{g}$. By definition,

$$
\partial_{\mathrm{s}} \tilde{\mathbf{g}}^{t}(s)=\mathbf{g}^{t}(s), \quad \partial_{t} \tilde{\mathbf{g}}^{t}(s)=\mathbf{g}(t)-\mathbf{g}^{t}(s)
$$

An integration by parts gives

$$
\int_{0}^{\infty} h(s) \mathbf{g}^{t}(s) \mathrm{d} s=-\int_{0}^{\infty} h^{\prime}(s) \tilde{\mathbf{g}}^{t}(s) \mathrm{d} s
$$

Hence, we can write (28) as

$$
\mathbf{q}(t)=-k(\theta(t))\left[\mathbf{g}(t)-\int_{0}^{\infty} h^{\prime}(s) \tilde{\mathbf{g}}^{t}(s) \mathrm{d} s\right]
$$

The balance of energy (9) then becomes

$$
\begin{aligned}
\dot{e} & -\dot{F}(\varphi)-\frac{\theta}{\theta_{c}} \dot{G}(\varphi)-\gamma \dot{\varphi}^{2}-v \partial_{t} \frac{1}{2}|\nabla \varphi|^{2} \\
& =k \Delta \theta+k^{\prime}|\mathbf{g}|^{2}-k \nabla \cdot \int_{0}^{\infty} h^{\prime}(s) \tilde{\mathbf{g}}^{t}(s) \mathrm{d} s-k^{\prime} \mathbf{g} \cdot \int_{0}^{\infty} h^{\prime}(s) \tilde{\mathbf{g}}^{t}(s) \mathrm{d} s+r
\end{aligned}
$$

the dependence on the present time $t$ being understood. Once we specify the form of the internal energy $e$, Equation (30) results in an evolution equation coupled with the Ginzburg-Landau equation (8).

\subsection{Thermodynamic potentials}

By (29) we can write

$$
\mathbf{q}(t)=-k(\theta(t))\left[\mathbf{g}(t)+\mathbf{w}\left(\tilde{\mathbf{g}}^{t}\right)\right]
$$

where the functional $\mathbf{w}\left(\tilde{\mathbf{g}}^{t}\right)$ is defined by

$$
\mathbf{w}\left(\tilde{\mathbf{g}}^{t}\right)=-\int_{0}^{\infty} h^{\prime}(s) \tilde{\mathbf{g}}^{t}(s) \mathrm{d} s
$$

For definiteness, we now choose particular forms of the free energy and examine the corresponding thermodynamic restrictions associated with the functional dependence (31).

Let the free energy depend on the present value of $\theta, \varphi, \nabla \varphi$ and on the summed history $\tilde{\mathbf{g}}^{t}$, which means that we regard $s=(\theta, \varphi, \nabla \varphi, \tilde{\mathbf{g}})$ as the state. The dependence on $s$ is assumed in the additive form:

$$
\psi(t)=\bar{\psi}(\theta(t), \varphi(t), \nabla \varphi(t))+\Psi\left(\tilde{\mathbf{g}}^{t}\right)
$$

where $\bar{\psi}$ and $\Psi$ are non-negative and subject to

$$
\bar{\psi}(0,0,0)=0, \quad \Psi(0)=0
$$


If $\mathbf{g}(\cdot)$ is a zero function, then (12) becomes

$$
\dot{\bar{\psi}}+\eta \dot{\theta}-\kappa \dot{\varphi}-\mathbf{p} \cdot \nabla \dot{\varphi} \leqslant 0
$$

Accordingly, the relations

$$
\eta=-\bar{\psi}_{\theta}, \quad v \nabla \varphi=\bar{\psi}_{\nabla \varphi}, \quad f+\frac{\theta}{\theta_{c}} g=\bar{\psi}_{\varphi}
$$

are required to hold. As a consequence, (12) reduces to

$$
\partial_{t} \Psi\left(\tilde{\mathbf{g}}^{t}\right)+\frac{1}{\theta(t)} \mathbf{q}(t) \cdot \mathbf{g}(t) \leqslant 0
$$

If $\Psi$ is differentiable, then

$$
\partial_{t} \Psi\left(\tilde{\mathbf{g}}^{t}\right)=\delta \Psi\left(\tilde{\mathbf{g}}^{t} \mid \mathbf{g}(t)-\mathbf{g}^{t}\right)
$$

$\delta$ denoting the Fréchet differential. By the linearity of the differential, we can write

$$
\partial_{t} \Psi\left(\tilde{\mathbf{g}}^{t}\right)=\mathbf{J}_{\mathbf{g}}\left(\tilde{\mathbf{g}}^{t}\right) \cdot \mathbf{g}(t)-\delta \Psi\left(\tilde{\mathbf{g}}^{t} \mid \mathbf{g}^{t}\right)
$$

where $\mathbf{J}_{\mathbf{g}}$ is the operator defined by

$$
\mathbf{J}_{\mathbf{g}}\left(\tilde{\mathbf{g}}^{t}\right) \cdot \mathbf{g}(t)=\delta \Psi\left(\tilde{\mathbf{g}}^{t} \mid \mathbf{g}(t)\right)
$$

Hence, (32) gives

$$
\left[\mathbf{J}_{\mathbf{g}}\left(\tilde{\mathbf{g}}^{t}\right)-\frac{k}{\theta}(t) \mathbf{w}\left(\tilde{\mathbf{g}}^{t}\right)\right] \cdot \mathbf{g}(t)-\delta \Psi\left(\tilde{\mathbf{g}}^{t} \mid \mathbf{g}^{t}\right)-\frac{k}{\theta}(t) \mathbf{g}^{2}(t) \leqslant 0
$$

Let $\mathbf{J}_{\mathbf{g}}$ and $\delta \Psi$ be continuous functionals. We can arbitrarily change $\mathbf{g}(t)$ and meanwhile the change of $\mathbf{J}_{\mathbf{g}}\left(\tilde{\mathbf{g}}^{t}\right), \delta \Psi\left(\tilde{\mathbf{g}}^{t} \mid \mathbf{g}^{t}\right)$ and $\mathbf{w}\left(\tilde{\mathbf{g}}^{t}\right)$ can be as small as we choose. This implies that (33) holds only if

$$
\left[\mathbf{J}_{\mathbf{g}}\left(\tilde{\mathbf{g}}^{t}\right)-\frac{k}{\theta}(t) \mathbf{w}\left(\tilde{\mathbf{g}}^{t}\right)\right] \cdot \mathbf{g}(t)-\frac{k}{\theta}(t) \mathbf{g}^{2}(t) \leqslant 0, \quad \delta \Psi\left(\tilde{\mathbf{g}}^{t} \mid \mathbf{g}^{t}\right) \geqslant 0
$$

Moreover, the arbitrariness of $\mathbf{g}(t)$ implies that

$$
\mathbf{J}_{\mathbf{g}}\left(\tilde{\mathbf{g}}^{t}\right)-\frac{k}{\theta}(t) \mathbf{w}\left(\tilde{\mathbf{g}}^{t}\right)=0, \quad k \geqslant 0
$$

Conversely, (34) and

$$
\delta \Psi\left(\tilde{\mathbf{g}}^{t} \mid \mathbf{g}^{t}\right) \geqslant 0
$$

are sufficient for the validity of (33) and hence of (32).

It follows from (34) that $k / \theta$ has to be independent of $\theta$. As a consequence, assuming that $\Psi$ is a functional on $\tilde{\mathbf{g}}^{t}$ only amounts to letting $k / \theta$ be a constant, namely

$$
k(\theta)=k_{0} \theta
$$


By (34), we have

$$
\mathbf{w}\left(\tilde{\mathbf{g}}^{t}\right)=\frac{1}{k_{0}} \mathbf{J}_{\mathbf{g}}\left(\tilde{\mathbf{g}}^{t}\right)
$$

which shows that the history-dependent heat flux is determined by $\delta \Psi$. The inequality in (35) is a restriction on the dissipation part.

If the transition temperature is much smaller than $\theta_{\mathrm{D}}$, then it is reasonable to assume that

$$
e_{\theta}=c \theta, \quad c>0
$$

We can then represent the functionals for free energy, entropy and internal energy in the form:

$$
\begin{aligned}
\psi & =-\frac{1}{2} c \theta^{2}+F(\varphi)+\frac{\theta}{\theta_{c}} G(\varphi)+\frac{1}{2} v|\nabla \varphi|^{2}+\Psi\left(\tilde{\mathbf{g}}^{t}\right) \\
\eta & =c \theta-\frac{1}{\theta_{c}} G(\varphi) \\
e & =F(\varphi)+\frac{1}{2} v|\nabla \varphi|^{2}+\frac{1}{2} c \theta^{2}+\Psi\left(\tilde{\mathbf{g}}^{t}\right)
\end{aligned}
$$

If, instead, the transition temperature is larger than $\theta_{\mathrm{D}}$, then we regard the specific heat as a constant, $c$. Accordingly, we take the free energy in the form

$$
\psi=-c \theta \ln \theta+F(\varphi)+\frac{\theta}{\theta_{c}} G(\varphi)+\frac{1}{2} v|\nabla \varphi|^{2}+\Psi\left(\tilde{\mathbf{g}}^{t}\right)
$$

whence we have

$$
\begin{aligned}
& \eta=c(1+\ln \theta) \theta-\frac{1}{\theta_{c}} G(\varphi) \\
& e=F(\varphi)+\frac{1}{2} v|\nabla \varphi|^{2}+c \theta+\Psi\left(\tilde{\mathbf{g}}^{t}\right)
\end{aligned}
$$

\section{FREE ENERGIES FOR THE SUMMED HISTORIES}

We look again at the constitutive equation (29) for q. Since

$$
\frac{\mathrm{d}}{\mathrm{d} t} \Psi\left(\tilde{\mathbf{g}}^{t}\right)=k_{0} \mathbf{w}\left(\tilde{\mathbf{g}}^{t}\right) \cdot \mathbf{g}(t)-\delta \Psi\left(\tilde{\mathbf{g}}^{t} \mid \mathbf{g}^{t}\right)
$$

by (35) it follows that

$$
\frac{\mathrm{d}}{\mathrm{d} t} \Psi\left(\tilde{\mathbf{g}}^{t}\right) \leqslant k_{0} \mathbf{w}\left(\tilde{\mathbf{g}}^{t}\right) \cdot \mathbf{g}(t)
$$

We now ascertain that there are functionals satisfying (38).

First, we consider the maximum free energy $\Psi_{\mathrm{M}}$, analogous to that for linear viscoelasticity [16], in the form:

$$
\Psi_{\mathrm{M}}\left(\tilde{\mathbf{g}}^{t}\right)=\frac{1}{2} k_{0} \int_{0}^{\infty} \int_{0}^{\infty} h^{\prime \prime}\left(\left|s_{1}-s_{2}\right|\right) \tilde{\mathbf{g}}^{t}\left(s_{1}\right) \cdot \tilde{\mathbf{g}}^{t}\left(s_{2}\right) \mathrm{d} s_{1} \mathrm{~d} s_{2}
$$


where $h$ is subject to

$$
\int_{0}^{\infty} h(\xi) \cos \omega \xi \mathrm{d} \xi \geqslant 0
$$

Hence, $\Psi_{M}$ proves to be non-negative. We denote by $D_{M}$ the domain of $\Psi_{M}$ namely

$$
D_{\mathrm{M}}=\left\{\tilde{\mathbf{g}}^{t}: \Psi_{\mathrm{M}}\left(\tilde{\mathbf{g}}^{t}\right)<\infty\right\}
$$

A direct calculation shows that (38) holds as an equality:

$$
\partial_{t} \Psi_{\mathrm{M}}\left(\tilde{\mathbf{g}}^{t}\right)=k_{0} \mathbf{w}\left(\tilde{\mathbf{g}}^{t}\right) \cdot \mathbf{g}(t)
$$

The maximum property of $\Psi_{\mathrm{M}}$ is characterized by

$$
\Psi_{\mathrm{M}}\left(\tilde{\mathbf{g}}^{t}\right) \geqslant \Psi\left(\tilde{\mathbf{g}}^{t}\right) \quad \forall \tilde{\mathbf{g}}^{t} \in D_{\mathrm{M}}
$$

A second functional, $\Psi_{\mathrm{G}}$, is the analogue of the Graffi-Volterra free energy in linear viscoelasticity. It is given by

$$
\Psi_{\mathrm{G}}\left(\tilde{\mathbf{g}}^{t}\right)=-\frac{k_{0}}{2} \int_{0}^{\infty} h^{\prime}(s) \tilde{\mathbf{g}}^{t}(s) \cdot \tilde{\mathbf{g}}^{t}(s) \mathrm{d} s
$$

where $h \in C^{2}\left(\mathbb{R}^{+}\right)$is subject to

$$
h^{\prime}(s)<0, \quad h^{\prime \prime}(s) \geqslant 0
$$

and hence satisfies (39). We denote by $D_{\mathrm{G}}$ the domain of definition:

$$
D_{\mathrm{G}}=\left\{\tilde{\mathbf{g}}^{t}: \Psi_{\mathrm{G}}\left(\tilde{\mathbf{g}}^{t}\right)<\infty\right\}
$$

As proved in [17], along the lines of [18], if

$$
\alpha h^{\prime}(s)+h^{\prime \prime}(s) \geqslant 0, \quad s \in \mathbb{R}^{+}
$$

for some $\alpha \geqslant 0$, then

$$
\partial_{t} \Psi_{\mathrm{G}}\left(\tilde{\mathbf{g}}^{t}\right) \leqslant-\alpha \Psi_{\mathrm{G}}\left(\tilde{\mathbf{g}}^{t}\right)+k_{0} \mathbf{w}\left(\tilde{\mathbf{g}}^{t}\right) \cdot \mathbf{g}(t)
$$

It is easy to check that

$$
\delta \Psi_{\mathrm{G}}\left(\tilde{\mathbf{g}}^{t} \mid \mathbf{g}^{t}\right)=-k_{0} \int_{0}^{\infty} h^{\prime}(s) \tilde{\mathbf{g}}^{t}(s) \cdot \mathbf{g}^{t}(s) \mathrm{d} s=\frac{k_{0}}{2} \int_{0}^{\infty} h^{\prime \prime}(s) \tilde{\mathbf{g}}^{t}(s) \cdot \tilde{\mathbf{g}}^{t}(s) \mathrm{d} s \geqslant 0
$$

whence (38) holds.

A further free energy, which is the minimum one, $\Psi_{\mathrm{m}}$ is considered in [19] borrowing from the Golden representation of the free energy for viscoelastic materials [20] (see [21]).

A free energy functional, which proves convenient for applications, arises from a different description of the fading memory. Hence, we let $t=t_{0}+\tau$, regard $t_{0}$ as an initial time, possibly $t_{0}=0$. Based upon an integration by parts, we can write

$$
\mathbf{w}\left(\tilde{\mathbf{g}}^{t_{0}+\tau}\right)=\int_{0}^{\infty} h(s) \mathbf{g}^{t_{0}+\tau}(s) \mathrm{d} s
$$


Now,

$$
\begin{aligned}
\int_{0}^{\infty} h(s) \mathbf{g}^{t_{0}+\tau}(s) \mathrm{d} s & =\int_{0}^{\tau} h(s) \mathbf{g}^{t_{0}+\tau}(s) \mathrm{d} s+\int_{\tau}^{\infty} h(s) \mathbf{g}^{t_{0}+\tau}(s) \mathrm{d} s \\
& =\int_{0}^{\tau} h(s) \mathbf{g}^{t_{0}+\tau}(s) \mathrm{d} s+\int_{0}^{\infty} h(\tau+\xi) \mathbf{g}^{t_{0}}(\xi) \mathrm{d} \xi
\end{aligned}
$$

and hence a further integration by parts gives

$$
\mathbf{w}\left(\tilde{\mathbf{g}}^{t_{0}+\tau}\right)=\int_{0}^{\tau} h(s) \mathbf{g}^{t_{0}+\tau}(s) \mathrm{d} s-\mathbf{I}^{t_{0}}(\tau)
$$

where

$$
\mathbf{I}^{t_{0}}(\tau):=\int_{0}^{\infty} h^{\prime}(\tau+\xi) \tilde{\mathbf{g}}^{t_{0}}(\xi) \mathrm{d} \xi
$$

The conceptual and operative meaning of (43) is apparent. For any function $\mathbf{g}$, we have

$$
\mathbf{w}\left(\tilde{\mathbf{g}}^{t_{0}}\right)=-\mathbf{I}^{t_{0}}(0)
$$

Also, if $\mathbf{g}=0$ in $\left(t_{0}, t_{0}+\tau\right)$ then

$$
\mathbf{w}\left(\tilde{\mathbf{g}}^{t_{0}+\tau}\right)=-\mathbf{I}^{t_{0}}(\tau)
$$

This means that $-\mathbf{I}^{t_{0}}(0)$ is the initial value of $\mathbf{w}$ at time $t_{0}$ and, moreover, $-\mathbf{I}^{t_{0}}(\tau)$ is the value of $\mathbf{w}$ at time $t_{0}+\tau$ if $\mathbf{g}$ vanishes in $\left(t_{0}, t_{0}+\tau\right)$. By (43) the response of the material, at $t_{0}+\tau$, is given by $\mathbf{I}^{t_{0}}(\tau)$ and the function $\mathbf{g}$ on $\left[t_{0}, t_{0}+\tau\right)$. Hence, following the lines of [22-24], we regard the state at time $t_{0}+\tau$ as the pair of $\mathbf{I}^{t}(\tau)$ and the process $\mathbf{g}$ on $\left[t_{0}, t_{0}+\tau\right)$. This is because the state is required for determining a unique response of the material to a given process.

By

$$
\mathbf{I}^{t}(\tau)=\int_{0}^{\infty} h^{\prime}(\tau+\xi) \tilde{\mathbf{g}}^{t}(\xi) \mathrm{d} \xi=-\int_{0}^{\infty} h(\tau+\xi) \mathbf{g}^{t}(\xi) \mathrm{d} \xi
$$

we can view $\mathbf{I}^{t}$ as determined by the summed history $\tilde{\mathbf{g}}^{t}$ or by $\mathbf{g}^{t}$. Moreover, we have

$$
\mathbf{w}\left(\tilde{\mathbf{g}}^{t}\right)=-\mathbf{I}^{t}(0)
$$

Also we can define the functional $\Psi_{\mathrm{I}}$ on $\mathbf{I}^{t}$ in the form

$$
\Psi_{\mathrm{I}}\left(\mathbf{I}^{t}\right)=-\frac{k_{0}}{2} \int_{0}^{\infty}\left[h^{\prime}(\tau)\right]^{-1} \partial_{\tau} \mathbf{I}^{t}(\tau) \cdot \partial_{\tau} \mathbf{I}^{t}(\tau) \mathrm{d} \tau
$$

We now show that $\Psi_{\mathrm{I}}$ is a free energy.

A direct evaluation shows that

$$
\partial_{t} \mathbf{I}^{t}(\tau)=-h(\tau) \mathbf{g}(t)+\partial_{\tau} \mathbf{I}^{t}(\tau)
$$


By using the exchange of the order of differentiation, identity (45) and an integration by parts we obtain

$$
\dot{\psi}_{\mathrm{I}}\left(\mathbf{I}^{t}\right)=k_{0} \mathbf{g}(t) \cdot \mathbf{w}\left(\tilde{\mathbf{g}}^{t}\right)-\frac{k_{0}}{2}\left[\frac{1}{h^{\prime}(\tau)} \partial_{\tau} \mathbf{I}^{t}(\tau) \cdot \partial_{\tau} \mathbf{I}^{t}(\tau)\right]_{0}^{\infty}-\Phi_{\mathrm{I}}\left(\mathbf{I}^{t}\right)
$$

where

$$
\Phi_{\mathrm{I}}\left(\mathbf{I}^{t}\right)=\frac{k_{0}}{2} \int_{0}^{\infty} \frac{h^{\prime \prime}(\tau)}{\left[h^{\prime}(\tau)\right]^{2}} \partial_{\tau} \mathbf{I}^{t}(\tau) \cdot \partial_{\tau} \mathbf{I}^{t}(\tau) \mathrm{d} \tau
$$

Because of the assumption $h^{\prime \prime} \geqslant 0$, we have

$$
\Phi_{\mathrm{I}}\left(\mathbf{I}^{t}\right) \geqslant 0
$$

Let $\mu=-h^{\prime}$ and hence $\mu>0$. Since

$$
\partial_{\tau} \mathbf{I}^{t}(\tau)=\int_{0}^{\infty} \mu(\tau+\xi) \mathbf{g}(t-\xi) \mathrm{d} \xi
$$

we can write

$$
\begin{aligned}
{\left[\frac{1}{h^{\prime}(\tau)} \partial_{\tau} \mathbf{I}^{t}(\tau) \cdot \partial_{\tau} \mathbf{I}^{t}(\tau)\right]_{0}^{\infty} } & =-\lim _{\tau \rightarrow \infty}\left[\int_{0}^{\infty} \frac{\mu(\tau+\xi)}{\sqrt{\mu(\tau)}} \mathbf{g}^{t}(\xi) \mathrm{d} \xi\right]^{2}+\frac{1}{\mu(0)}\left[\int_{0}^{\infty} \mu(\xi) \mathbf{g}^{t}(\xi) \mathrm{d} \xi\right]^{2} \\
& \geqslant-\lim _{\tau \rightarrow \infty}\left[\int_{0}^{\infty} \frac{\mu^{2}(\tau+\xi)}{\mu(\tau) \mu(\xi)} \mathrm{d} \xi\right] \int_{0}^{\infty} \mu(\xi)\left|\mathbf{g}^{t}(\xi)\right|^{2} \mathrm{~d} \xi
\end{aligned}
$$

Provided that

$$
\int_{0}^{\infty} \mu(\xi)\left|\mathbf{g}^{t}(\xi)\right|^{2} \mathrm{~d} \xi<\infty
$$

and

$$
\lim _{\tau \rightarrow \infty} \int_{0}^{\infty} \frac{\mu^{2}(\tau+\xi)}{\mu(\tau) \mu(\xi)} \mathrm{d} \xi=0
$$

we conclude that

$$
\left[\frac{1}{h^{\prime}(\tau)} \partial_{\tau} \mathbf{I}^{t}(\tau) \cdot \partial_{\tau} \mathbf{I}^{t}(\tau)\right]_{0}^{\infty} \geqslant 0
$$

By (46) and (48) we find that

$$
\dot{\psi}_{\mathrm{I}}\left(\mathbf{I}^{t}\right) \leqslant k_{0} \mathbf{g}(t) \cdot \mathbf{w}\left(\tilde{\mathbf{g}}^{t}\right)
$$

and hence conclude that $\Psi_{\mathrm{I}}$ is a free-energy functional.

To ascertain that (47) may hold, we consider a function $\mu$ such that

$$
\mu(\tau+\xi) \leqslant f(\tau) \mu(\xi) \quad \forall \tau, \xi \geqslant 0
$$


where $f$ is subject to

$$
f(\tau)>0, \quad f(0) \geqslant 1, \quad \lim _{\tau \rightarrow \infty} f(\tau)=0, \quad \int_{0}^{\infty} f(\xi) \mathrm{d} \xi=F<\infty
$$

The trivial integration gives

$$
\int_{0}^{\infty} \frac{\mu^{2}(\tau+\xi)}{\mu(\tau) \mu(\xi)} \mathrm{d} \xi \leqslant \int_{0}^{\infty} f(\tau) f(\xi) \mathrm{d} \xi=F f(\tau)
$$

whence (47) follows. In particular, $f(\tau)=\exp (-\alpha \tau)$ may be considered and, in such a case, the condition (49) is equivalent to (41).

By (48), we can write

$$
\dot{\psi}_{\mathrm{I}}\left(\mathbf{I}^{t}\right) \leqslant k_{0} \mathbf{w}\left(\tilde{\mathbf{g}}^{t}\right) \cdot \mathbf{g}(t)-\Phi_{\mathrm{I}}\left(\mathbf{I}^{t}\right)
$$

If (41), we have

$$
0 \leqslant \frac{h^{\prime \prime}}{\left[h^{\prime}\right]^{2}}+\alpha \frac{h^{\prime}}{\left[h^{\prime}\right]^{2}}=\frac{h^{\prime \prime}}{\left[h^{\prime}\right]^{2}}+\alpha\left[h^{\prime}\right]^{-1}
$$

Hence, we have

$$
\Phi_{\mathrm{I}} \geqslant \alpha \Psi_{\mathrm{I}}
$$

whence

$$
\dot{\psi}_{\mathrm{I}}\left(\mathbf{I}^{t}\right) \leqslant k_{0} \mathbf{w}\left(\tilde{\mathbf{g}}^{t}\right) \cdot \mathbf{g}(t)-\alpha \Psi_{\mathrm{I}}\left(\mathbf{I}^{t}\right)
$$

The set of free energies is convex and the domains of the free-energy functionals $\mathscr{D} \Psi$ depend on the functional under consideration. Indeed, we can show that

$$
\mathscr{D} \Psi_{\mathrm{M}} \subset \mathscr{D} \Psi_{\mathrm{G}} \subset \mathscr{D} \Psi_{\mathrm{I}} \subset \mathscr{D} \Psi_{\mathrm{m}}
$$

and the values of the functionals are related by

$$
\Psi_{\mathrm{m}}\left(\tilde{\mathbf{g}}^{t}\right) \leqslant \Psi_{\mathrm{I}}\left(\mathbf{I}^{t}\right) \leqslant \Psi_{\mathrm{G}}\left(\tilde{\mathbf{g}}^{t}\right) \leqslant \Psi_{\mathrm{M}}\left(\tilde{\mathbf{g}}^{t}\right)
$$

With any free energy $\Psi$, we can associate a seminorm in $\mathscr{D} \Psi$ by

$$
\left\|\tilde{\mathbf{g}}^{t}\right\|^{2}=\frac{2}{k_{0}} \Psi\left(\tilde{\mathbf{g}}^{t}\right)
$$

Moreover, the constitutive functional (29) for $\mathbf{q}$ is continuous relative to the seminorm (50).

As with any system with memory, the state is infinite dimensional and the associated stability properties may depend on the selected norm. It follows from definition (50) that if

$$
\Psi_{a}\left(\tilde{\mathbf{g}}^{t}\right) \leqslant \Psi_{b}\left(\tilde{\mathbf{g}}^{t}\right)
$$

then

$$
\mathscr{D} \Psi_{b} \subseteq \mathscr{D} \Psi_{a}
$$


Hence, if the system is stable relative to the $\Psi_{b}$-norm then it is stable also relative to the $\Psi_{a}$-norm. The converse is not true.

With this in mind, proving stability properties relative to $\Psi_{\mathrm{I}}$ is convenient in two respects. First, stability holds in a domain, $\mathscr{D}_{\mathrm{I}}$, which is larger than those for $\Psi_{\mathrm{G}}$ and $\Psi_{\mathrm{M}}$. Secondly, $\Psi_{\mathrm{I}}$ is defined on $\mathbf{I}^{t}$, which is the minimal state of the system.

\section{EVOLUTION PROBLEMS IN CONDUCTORS WITH MEMORY}

In this section, we investigate the system of differential equations, for a first-order phase transition, for a heat conductor with memory modelled by $(28)$, in a smooth three-dimensional region $\Omega$. Upon substitution of $e$ from (36) into the balance equation (30), and observing that

$$
\frac{\mathrm{d}}{\mathrm{d} t} \Psi\left(\tilde{\mathbf{g}}^{t}\right)=\frac{k}{\theta}(t) \mathbf{w}\left(\tilde{\mathbf{g}}^{t}\right) \cdot \mathbf{g}(t)-\delta \Psi\left(\tilde{\mathbf{g}}^{t} \mid \mathbf{g}^{t}\right)
$$

we have

$$
c \dot{\theta}-\delta \Psi\left(\tilde{\mathbf{g}}^{t} \mid \mathbf{g}^{t}\right)-\frac{\theta}{\theta_{c}} g(\varphi) \dot{\varphi}-\gamma \dot{\varphi}^{2}=k(\theta) \Delta \theta+k^{\prime}(\theta)|\mathbf{g}|^{2}+k(\theta) \nabla \cdot \mathbf{w}\left(\tilde{\mathbf{g}}^{t}\right)+r
$$

Again we let $k / \theta$ be a constant, $k_{0}$. Moreover, having in mind evolutions near equilibrium $(\mathbf{g}=0, \dot{\varphi}=0)$, we make the approximation that $\dot{\varphi}^{2}$ and $|\nabla \theta|^{2}$ are negligible. With appropriate assumptions on $h$, at least for $\Psi_{\mathrm{M}}, \Psi_{\mathrm{G}}, \Psi_{\mathrm{I}}$, we can show that

$$
\delta \Psi\left(\tilde{\mathbf{g}}^{t} \mid \mathbf{g}^{t}\right) \leqslant C(h) \sup _{s \in[0, \infty)}\left|\mathbf{g}^{t}(s)\right|^{2}
$$

thus proving that $\delta \Psi$ is negligible if so is $|\mathbf{g}(t)|^{2}$ at any time $t>0$. Hence, upon dividing throughout by $\theta$, we can write the approximate version of (55) as

$$
\frac{c}{\theta} \dot{\theta}-\frac{1}{\theta_{c}} g(\varphi) \dot{\varphi}=k_{0} \Delta \theta+k_{0} \nabla \cdot \mathbf{w}\left(\tilde{\mathbf{g}}^{t}\right)+\frac{r}{\theta}
$$

whence

$$
\partial_{t}\left[c \ln \theta-\frac{1}{\theta_{c}} G(\varphi)\right]=k_{0} \Delta \theta+k_{0} \nabla \cdot \int_{0}^{\infty} h(s) \nabla \theta(t-s) \mathrm{d} s+\frac{r}{\theta}
$$

where $g=G^{\prime}$. In the terminology of, e.g. [4] (see also [25]), Equation (59) would be referred to as the entropy equation. It has to be associated with the Ginzburg-Landau equation (7), within the evolution problem, as follows.

\section{Problem 1}

The past history of $\theta$ up to $t=0$ is known, $\theta(-s)=\hat{\theta}(s), s>0$. We denote the known function by $R$

$$
R(t)=\frac{r(t)}{\theta(t)}+k_{0} \nabla \cdot \int_{0}^{\infty} h(t+\xi) \nabla \hat{\theta}(\xi) \mathrm{d} \xi
$$


The state of the material at time $t$ is given by the pair $(\theta(t), \varphi(t))$. The state evolution is governed by the system of differential equations

$$
\begin{aligned}
\partial_{t}\left[c \ln \theta-\frac{1}{\theta_{c}} G(\varphi)\right] & =k_{0} \Delta \theta+k_{0} \nabla \cdot \int_{0}^{t} \nabla \theta(t-s) \mathrm{d} s+R \\
\gamma \partial_{t} \varphi+f(\varphi)+\frac{\theta}{\theta_{c}} g(\varphi) & =v \Delta \varphi
\end{aligned}
$$

subject to the boundary and initial conditions

$$
\begin{aligned}
\theta & =\theta_{\partial \Omega}, \quad \nabla \varphi \cdot \mathbf{n}=0 \text { on } \partial \Omega \\
\theta(0) & =\theta_{0}, \quad \varphi(0)=\varphi_{0} \text { in } \Omega
\end{aligned}
$$

Heretofore, the dependence on the position $\mathbf{x} \in \Omega$ is understood and not written.

\subsection{Evolution equations in the history-space setting}

We now set up a different scheme by viewing $\tilde{\mathbf{g}}^{t}$ as a part of the state that accounts for the history of $\mathbf{g}$ up to time $t$. This is made possible by the feature that different summed histories $\tilde{\mathbf{g}}^{t}$ produce different response functions $\mathbf{I}^{t}$.

The summed history $\tilde{\mathbf{g}}^{t}$ satisfies a differential equation of the transport type,

$$
\partial_{t} \tilde{\mathbf{g}}^{t}(s)+\partial_{s} \tilde{\mathbf{g}}^{t}(s)=\mathbf{g}(t)
$$

and the boundary condition

$$
\tilde{\mathbf{g}}^{t}(0)=0
$$

Since (52) can be written in the form

$$
\nabla \cdot \mathbf{w}\left(\tilde{\mathbf{g}}^{t}\right)=k_{0} \nabla \cdot \int_{0}^{\infty} \mu(s) \tilde{\mathbf{g}}^{t}(s) \mathrm{d} s
$$

in view of (52), (7) and (62), we can state the evolution problem as follows.

\section{Problem 2}

Represent the state of the material, at time $t$, by the triplet $\left(\theta(t), \varphi(t), \tilde{\mathbf{g}}^{t}\right)$. Hence, the evolution of the state is governed by the system of differential equations:

$$
\begin{aligned}
\partial_{t}\left[c \ln \theta-\frac{1}{\theta_{c}} G(\varphi)\right] & =k_{0} \Delta \theta+k_{0} \nabla \cdot \int_{0}^{\infty} \mu(s) \tilde{\mathbf{g}}^{t}(s) \mathrm{d} s+\frac{r}{\theta} \\
\gamma \partial_{t} \varphi+f(\varphi)+\frac{\theta}{\theta_{c}} g(\varphi) & =v \Delta \varphi \\
\partial_{t} \tilde{\mathbf{g}}^{t}(s) & =\nabla \theta(t)-\partial_{s} \tilde{\mathbf{g}}^{t}(s)
\end{aligned}
$$

subject to the boundary conditions

$$
\theta=\theta_{\partial \Omega}, \quad \nabla \varphi \cdot \mathbf{n}=0 \text { on } \partial \Omega, \quad \tilde{\mathbf{g}}^{t}(0)=0 \text { in } \Omega
$$


and the initial conditions

$$
\theta(0)=\theta_{0}, \quad \varphi(0)=\varphi_{0}, \quad \tilde{\mathbf{g}}^{0}(s)=\mathbf{f}_{0}(s) \quad s>0 \text { in } \Omega
$$

In this setting, the history space is $L_{\mu}^{2}\left(\mathbb{R}, L^{2}(\Omega)\right)$ endowed with the natural norm:

$$
\left\|\tilde{\mathbf{g}}^{t}\right\|_{\mu}^{2}=\frac{2}{k_{0}} \int_{\Omega} \Psi_{\mathrm{G}}\left(\tilde{\mathbf{g}}^{t}\right) \mathrm{d} v=\int_{0}^{\infty} \mu(s) \int_{\Omega}\left|\tilde{\mathbf{g}}^{t}\right|^{2} \mathrm{~d} v \mathrm{~d} s
$$

If $r / \theta$ is a known function of the position $\mathbf{x}$ only then well posedness leads to the existence of a continuous semigroup of solutions.

\subsection{Evolution equations in the minimal-state space setting}

We now regard the material state component, which accounts for the past history of $\mathbf{g}$ up to time $t$, as represented by a class of different but equivalent functions $\tilde{\mathbf{g}}^{t}$ which does not reduce to a singleton. Indeed, different summed history functions $\tilde{\mathbf{g}}^{t}$ are defined to be equivalent if they produce the same response function $\mathbf{I}^{t}$. As a consequence, $\mathbf{I}^{t}$ is taken to represent all of the history functions in the same class, which realizes the minimal state representation. Hence, we write the constitutive equation (29) for $\mathbf{q}$, in terms of $\mathbf{I}^{t}(0), \mathbf{g}$ and $\theta$, in the form

$$
\mathbf{q}(t)=-k_{0} \theta(t)\left[\mathbf{g}(t)-\mathbf{I}^{t}(0)\right]
$$

and the free energy in the form (44). For later convenience, we observe that

$$
\mathbf{I}^{t}(\tau)=\int_{0}^{\infty} h^{\prime}(\tau+\xi) \tilde{\mathbf{g}}^{t}(\xi) \mathrm{d} \xi
$$

satisfies the transport-like equation

$$
\partial_{t} \mathbf{I}^{t}(\tau)=-h(\tau) \mathbf{g}(t)+\partial_{\tau} \mathbf{I}^{t}(\tau)
$$

Unlike the previous case, we now cannot say that $\mathbf{I}^{t}(0)=0$. Indeed,

$$
\mathbf{I}^{t}(0)=-\mathbf{w}\left(\tilde{\mathbf{g}}^{t}\right)
$$

provides $\mathbf{w}$ in terms of the minimal state representation $\mathbf{I}^{t}$.

The free energy $\Psi_{\mathrm{I}}$ in (44) is a quadratic functional of $\partial_{\tau} \mathbf{I}^{t}$, not of $\mathbf{I}^{t}$. Hence, to set up a natural norm, we look at

$$
\mathbf{J}^{t}(\tau):=\partial_{\tau} \mathbf{I}^{t}(\tau)=-\int_{0}^{\infty} \mu^{\prime}(\tau+\xi) \tilde{\mathbf{g}}^{t}(\xi) \mathrm{d} \xi=\int_{0}^{\infty} \mu(\tau+\xi) \mathbf{g}^{t}(\xi) \mathrm{d} \xi
$$

The free energy $\Psi_{\mathrm{I}}$ then takes the form

$$
\Psi_{\mathrm{I}}\left(\mathbf{J}^{t}\right)=\frac{k_{0}}{2} \int_{0}^{\infty}[\mu(\tau)]^{-1}\left|\mathbf{J}^{t}(\tau)\right|^{2} \mathrm{~d} \tau
$$

The function $\mathbf{J}^{t}$ satisfies the differential equation

$$
\partial_{t} \mathbf{J}^{t}(\tau)=\mu(\tau) \mathbf{g}(t)+\partial_{\tau} \mathbf{J}^{t}(\tau)
$$


while

$$
\mathbf{J}^{t}(0)=\int_{0}^{\infty} \mu(\xi) \mathbf{g}^{t}(\xi) \mathrm{d} \xi
$$

Because

$$
\mathbf{I}^{t}(0)=-\int_{0}^{\infty} \int_{0}^{\infty} \mu(s) \tilde{\mathbf{g}}^{t}(s) \mathrm{d} s=\int_{0}^{\infty} \int_{0}^{\infty} \mu^{\prime}(\tau+s) \mathrm{d} \tau \tilde{\mathbf{g}}^{t}(s) \mathrm{d} s
$$

and

$$
\mathbf{I}^{t}(0)=-\mathbf{w}\left(\tilde{\mathbf{g}}^{t}\right)=-\int_{0}^{\infty} \mathbf{J}^{t}(\tau) \mathrm{d} \tau
$$

it follows that the constitutive equation (28) for $\mathbf{q}$ can be given the form

$$
\mathbf{q}(t)=-k_{0} \theta(t)\left[\mathbf{g}(t)+\int_{0}^{\infty} \mathbf{J}^{t}(\tau) \mathrm{d} \tau\right]
$$

A direct calculation provides

$$
\dot{\psi}_{\mathrm{I}}\left(\mathbf{J}^{t}\right)=k_{0} \tilde{\mathbf{w}}\left(\mathbf{J}^{t}\right) \cdot \mathbf{g}(t)-\delta \Psi_{\mathrm{I}}\left(\mathbf{J}^{t} \mid \mathbf{g}^{t}\right)
$$

which is the analogue of (37). Indeed, provided $\mu^{\prime} \leqslant 0, \mu>0$, we find that

$$
\delta \Psi_{\mathrm{I}}\left(\mathbf{J}^{t} \mid \mathbf{g}^{t}\right)=-\frac{k_{0}}{2} \int_{0}^{\infty}[\mu(\tau)]^{-2} \mu^{\prime}(\tau)\left|\mathbf{J}^{t}(\tau)\right|^{2} \mathrm{~d} \tau+\frac{1}{\mu(0)}\left[\int_{0}^{\infty} \mu(\xi) \mathbf{g}^{t}(\xi) \mathrm{d} \xi\right]^{2} \geqslant 0
$$

and hence

$$
\dot{\psi}_{\mathrm{I}}\left(\mathbf{J}^{t}\right) \leqslant k_{0} \tilde{\mathbf{w}}\left(\mathbf{J}^{t}\right) \cdot \mathbf{g}(t)
$$

These relations allow us to state the evolution problem as follows.

\section{Problem 3}

The state of the material at time $t$ is the triplet $\left(\theta(t), \varphi(t), \mathbf{J}^{t}\right)$. The state evolution is governed by the system of differential equations

$$
\begin{aligned}
\partial_{t}\left[c \ln \theta-\frac{1}{\theta_{c}} G(\varphi)\right] & =k_{0} \Delta \theta+k_{0} \nabla \cdot \int_{0}^{\infty} \mathbf{J}^{t}(\tau) \mathrm{d} \tau+\frac{r}{\theta} \\
\gamma \partial_{t} \varphi+f(\varphi)+\frac{\theta}{\theta_{c}} g(\varphi) & =v \Delta \varphi \\
\partial_{t} \mathbf{J}^{t}(\tau) & =\mu(\tau) \nabla \theta(t)+\partial_{\tau} \mathbf{J}^{t}(\tau)
\end{aligned}
$$

subject to the boundary conditions

$$
\theta=\theta_{\partial \Omega}, \quad \nabla \varphi \cdot \mathbf{n}=0 \quad \text { on } \partial \Omega
$$

and the initial conditions

$$
\theta(0)=\theta_{0}, \quad \varphi(0)=\varphi_{0}, \quad \mathbf{J}^{0}(s)=\mathbf{J}_{0}(s), \quad s>0 \text { in } \Omega
$$


Let $\zeta=1 / \mu$. In the present minimal-state setting, the underlying function space is $L_{\zeta}^{2}\left(\mathbb{R}^{+}, L^{2}(\Omega)\right)$ endowed with the natural norm

$$
\left\|\mathbf{J}^{t}\right\|_{\zeta}^{2}=\frac{2}{k_{0}} \int_{\Omega} \Psi_{\mathrm{I}}\left(\mathbf{J}^{t}\right) \mathrm{d} v=\int_{0}^{\infty} \zeta(s) \int_{\Omega}\left|\mathbf{J}^{t}(s)\right|^{2} \mathrm{~d} v \mathrm{~d} s
$$

If the source term $r / \theta$ is a known function, independent of $t$, then well posedness leads to the existence of a continuous semigroup of solutions.

\subsection{Stability}

As remarked at the end of Section 8 , it is convenient to prove stability with respect to the minimalstate space (Problem 3). To this end, we assume existence and uniqueness of the solution with positive temperature and enough regularity to guarantee the required a priori estimates. Well posedness of Problems 1-3 will be investigated in a future paper.

To investigate the stability of the solution to Problem 3 via standard methods, we need a more convenient formulation. First, we give evidence of the gradient form of the minimal-state variable $\mathbf{J}^{t}$ by means of the summed past history of the temperature,

$$
\tilde{\theta}^{t}(\tau)=\int_{0}^{\tau} \theta(t-s) \mathrm{d} s
$$

and the integral

$$
j^{t}(\tau)=-\int_{0}^{\infty} \mu^{\prime}(\tau+s) \tilde{\theta}^{t}(s) \mathrm{d} s=\int_{0}^{\infty} \mu(\tau+s) \theta^{t}(s) \mathrm{d} s
$$

Hence,

$$
\tilde{\mathbf{g}}^{t}(\tau)=\nabla \tilde{\theta}^{t}(\tau), \quad \mathbf{J}^{t}(\tau)=\nabla j^{t}(\tau)
$$

To make the boundary conditions homogeneous, we consider the function $\theta_{\mathscr{H}} \in H^{1}(\Omega)$ subject to

$$
\begin{array}{rlrl}
\Delta \theta_{\mathscr{H}} & =0 & & \text { in } \Omega \\
\theta_{\mathscr{H}}=\theta_{\partial \Omega} & & \text { on } \partial \Omega
\end{array}
$$

Let $\theta_{\partial \Omega}$ be time independent such that

$$
\theta_{\partial \Omega} \in H^{1 / 2}(\partial \Omega) \cap L^{\infty}(\partial \Omega), \quad 0<\theta_{*} \leqslant \theta_{\partial \Omega} \leqslant \theta^{*}
$$

Hence, $\theta_{\mathscr{H}}$ satisfies the inequalities

$$
\theta_{*} \leqslant \theta_{\mathscr{H}} \leqslant \theta^{*} \quad \text { a.e. in } \Omega \times(0, T)
$$

and

$$
\left\|\theta_{\mathscr{H}}\right\|_{H^{1}(\Omega)} \leqslant c\left\|\theta_{\partial \Omega}\right\|_{H^{1 / 2}(\partial \Omega)}
$$

The difference

$$
u=\theta-\theta_{\mathscr{H}}
$$


satisfies a homogeneous Dirichlet boundary condition. Moreover, $\Delta u=\Delta \theta$ identically. Accordingly, we introduce the new variable:

$$
\xi^{t}(\tau)=\int_{0}^{\infty} \mu(\tau+s) u^{t}(s) \mathrm{d} s=j^{t}(\tau)-h(\tau) \theta_{\mathscr{H}}, \quad h(\tau)=\int_{0}^{\infty} \mu(\tau+s) \mathrm{d} s
$$

It is easy to check that $\xi^{t}$ satisfies a homogeneous Dirichlet boundary condition and the identity $\Delta \xi^{t}=\Delta j^{t}$.

By means of the new scalar state variables $\left(u(t), \varphi(t), \xi^{t}\right)$, we can express Problem 3 by the evolution equations

$$
\begin{aligned}
\partial_{t}\left[c \ln \left(u+\theta_{\mathscr{H}}\right)-\frac{1}{\theta_{c}} G(\varphi)\right] & =k_{0} \Delta u+k_{0} \nabla \cdot \int_{0}^{\infty} \nabla \xi^{t}(s) \mathrm{d} s+\frac{r}{u+\theta_{\mathscr{H}}} \\
\gamma \partial_{t} \varphi+f(\varphi)+\frac{u+\theta_{\mathscr{H}}}{\theta_{c}} g(\varphi) & =v \Delta \varphi \\
\partial_{t} \xi^{t}(\tau) & =\mu(\tau) u(t)+\partial_{\tau} \xi^{t}(\tau)
\end{aligned}
$$

the homogeneous boundary conditions

$$
u(t)=0, \quad \nabla \varphi(t) \cdot \mathbf{n}=0, \quad \xi^{t}=0 \text { on } \partial \Omega
$$

and the initial conditions

$$
u(0)=\theta_{0}-\theta_{\mathscr{H}}, \quad \varphi(0)=\varphi_{0}, \quad \xi^{0}(\tau)=\xi_{0}(\tau), \quad \tau>0 \text { in } \Omega
$$

Also in view of (41), the data are required to satisfy the following assumptions.

$H_{1}: c, \gamma, v, \theta_{c}, k_{0}>0$

$H_{2}: \mu, \mu^{\prime} \in L^{1}\left(\mathbb{R}^{+}\right), \mu>0$ and $\mu^{\prime}+\alpha \mu \leqslant 0$ a.e. in $\mathbb{R}^{+}$;

$H_{3}: \varphi_{0} \in H^{1}(\Omega), 0 \leqslant \varphi_{0} \leqslant 1$ a.e. in $\Omega, \theta_{0} \in L^{\infty}(\Omega), \xi_{0} \in L_{\zeta}^{2}\left(\mathbb{R}^{+}, H^{1}(\Omega)\right)$;

$H_{4}: r \in L^{1}\left(\mathbb{R}^{+}, L^{\infty}(\Omega)\right)$;

$H_{5}$ : there exist two positive constants $\theta_{*}$ and $\theta^{*}$ such that $\theta_{*} \leqslant \theta_{0} \leqslant \theta^{*}$.

As usual, let $\|\cdot\|$ and $\langle\cdot, \cdot\rangle$ be the norm and the inner product of $L^{2}(\Omega)$. In addition, since $\zeta=1 / \mu$, let $\langle\cdot, \cdot\rangle_{\zeta}$ denote the inner product of $L_{\zeta}^{2}\left(\mathbb{R}^{+}, H^{1}(\Omega)\right)$. Also, $\Omega$ is assumed to be bounded and $|\Omega|$ denotes the volume. Assume the existence of a unique solution $\left(u(t), \varphi(t), \xi^{t}\right) \in L^{1}(\Omega) \times H^{1}(\Omega) \times$ $L_{\zeta}^{2}\left(\mathbb{R}^{+}, H^{1}(\Omega)\right)$ and take the energy norm of the system as

$$
\mathscr{E}(t)=\|u\|_{L^{1}(\Omega)}+\|\varphi\|_{H^{1}(\Omega)}^{2}+\left\|\xi^{t}\right\|_{\zeta}^{2}
$$

We now multiply (56), (57) by $u, \partial_{t} \varphi \in L^{2}(\Omega)$ and evaluate their inner products in $L^{2}(\Omega)$. Also, multiply (58) by $k_{0} \xi^{t}$ and evaluate the inner product in $L_{\zeta}^{2}\left(\mathbb{R}, H^{1}(\Omega)\right)$. Summation and the 
observation that $f=F^{\prime}, g=G^{\prime}$ yield

$$
\begin{gathered}
\gamma\left\|\partial_{t} \varphi\right\|^{2}+k_{0}\|\nabla u\|^{2}+c\left\langle\partial_{t} \theta, 1\right\rangle-c\left\langle\partial_{t} \ln \theta, \theta \mathscr{H}\right\rangle+\left\langle\partial_{t} F(\varphi), 1\right\rangle+v\left\langle\nabla \varphi, \partial_{t} \nabla \varphi\right\rangle \\
+\frac{1}{\theta_{c}}\left\langle\partial_{t} G(\varphi), \theta_{\mathscr{H}}\right\rangle+k_{0}\left\langle\xi^{t}, \partial_{t} \xi^{t}\right\rangle_{\zeta}=k_{0}\left\langle\partial_{\tau} \xi^{t}, \xi^{t}\right\rangle_{\zeta}+\langle r, 1\rangle-\left\langle r, \frac{\theta \mathscr{H}}{\theta}\right\rangle
\end{gathered}
$$

Since

$$
\left\langle\partial_{\tau} \xi^{t}, \xi^{t}\right\rangle_{\zeta}=-\frac{1}{2} \int_{0}^{\infty} \zeta^{\prime}(\tau)\left\|\nabla \xi^{t}(\tau)\right\|^{2} \mathrm{~d} \tau=\frac{1}{2} \int_{0}^{\infty} \frac{\mu^{\prime}(\tau)}{\mu^{2}(\tau)}\left\|\nabla \xi^{t}(\tau)\right\|^{2} \mathrm{~d} \tau \leqslant 0
$$

by means of the properties $H_{1}$ and $H_{2}$, we obtain the estimate

$$
\partial_{t}\left(c\langle\theta, 1\rangle-c\left\langle\ln \theta, \theta_{\mathscr{H}}\right\rangle+\langle F(\varphi), 1\rangle+\frac{1}{\theta_{c}}\left\langle G(\varphi), \theta_{\mathscr{H}}\right\rangle+\frac{v}{2}\|\nabla \varphi\|^{2}+\frac{k_{0}}{2}\left\|\xi^{t}\right\|_{\zeta}^{2}\right) \leqslant\left\langle r, 1-\frac{\theta_{\mathscr{H}}}{\theta}\right\rangle
$$

In view of (18) for $F$ and $G$, we consider the functions

$$
\begin{aligned}
& \Phi_{0}(t)=c\|\theta\|_{L^{1}(\Omega)}+3 L\|\varphi\|_{L^{4}(\Omega)}^{4}+\frac{v}{2}\|\nabla \varphi\|^{2}+\frac{k_{0}}{2}\left\|\xi^{t}\right\|_{\zeta}^{2} \\
& \Phi_{1}(t)=L \int_{\Omega}\left[3 \varphi^{4}-4\left(1+\frac{2 \theta_{\mathscr{H}}}{\theta_{c}}\right) \varphi^{3}+\alpha_{1}\right] \mathrm{d} v \\
& \Phi_{2}(t)=c \int_{\Omega}\left(\alpha_{2}-\theta_{\mathscr{H}} \ln \theta\right) \mathrm{d} v
\end{aligned}
$$

where $\alpha_{1}, \alpha_{2}$ are positive constants. By (60) the function

$$
\Phi(t)=\Phi_{0}(t)+\Phi_{1}(t)+\Phi_{2}(t)
$$

satisfies

$$
\dot{\Phi}(t) \leqslant\left\langle r, \frac{\theta-\theta_{\mathscr{H}}}{\theta}\right\rangle
$$

Incidentally, if $r=0$ we have $\dot{\Phi}(t) \leqslant 0$ and hence $\Phi(t) \leqslant \Phi(0)$.

We now assume that the initial value $\theta_{0}$ allows the temperature $\theta(t)$ to remain close to $\theta_{\mathscr{H}}$ in $\Omega$ for all times. This assumption is reasonable because $\theta(t)=\theta_{\mathscr{H}}$ on $\partial \Omega$ for all $t>0$. As a consequence, we make the approximation

$$
\left\langle\frac{r}{\theta}, \theta-\theta_{\mathscr{H}}\right\rangle \simeq\left\langle\frac{r}{\theta_{\mathscr{H}}}, \theta-\theta_{\mathscr{H}}\right\rangle
$$

Moreover, by means of (55) we can establish the estimate

$$
\left\langle\frac{r}{\theta_{\mathscr{H}}}, \theta-\theta_{\mathscr{H}}\right\rangle \leqslant \frac{1}{\theta}\left\|_{*} r(t)\right\|_{L^{\infty}(\Omega)}\left[\|\theta(t)\|_{L^{1}(\Omega)}+\theta^{*}|\Omega|\right] \leqslant \rho(t)\left[\Phi_{0}(t)+C\right]
$$

where

$$
C=c \theta^{*}|\Omega|, \quad \rho=\frac{1}{c \theta_{*}}\|r\|_{L^{\infty}(\Omega)} \in L^{1}\left(\mathbb{R}^{+}\right)
$$


Hence, by (61) we have

$$
\dot{\Phi}(t) \leqslant \rho(t)\left[\Phi_{0}(t)+C\right]
$$

To establish the boundedness of $\Phi_{0}$, we need a relation between $\Phi$ and $\Phi_{0}$. Provided we let $\alpha_{1}$ be sufficiently large and $\alpha_{0}$ be sufficiently small, we can write

$$
\int_{\Omega}\left[3 \varphi^{4}-4\left(1+\frac{2 \theta_{\mathscr{H}}}{\theta_{c}}\right) \varphi^{3}+\alpha_{1}\right] \mathrm{d} v \geqslant 3 \alpha_{0} \int_{\Omega} \frac{\theta_{\mathscr{H}}}{\theta_{c}} \varphi^{2}\left(\varphi^{2}+2\right) \mathrm{d} v>0
$$

Moreover, the inequality $\ln \theta \leqslant \sqrt{\theta}$ and the bounds (55) imply that, for a sufficiently large $\alpha_{2}$ and a sufficiently small $c_{0}<1$,

$$
\int_{\Omega}\left(\theta-\theta_{\mathscr{H}} \ln \theta+\alpha_{2}\right) \mathrm{d} v \geqslant \int_{\Omega}\left(\theta-\theta^{*} \sqrt{\theta}+\alpha_{2}\right) \mathrm{d} v \geqslant c_{0} \int_{\Omega} \theta \mathrm{d} v
$$

Collecting these results, we can write

$$
\Phi \geqslant \Phi_{0}+\Phi_{2} \geqslant c_{0} c\|\theta\|_{L^{1}(\Omega)}+3 L\|\varphi\|_{L^{4}(\Omega)}^{4}+\frac{v}{2}\|\nabla \varphi\|^{2}
$$

Hence, $\Phi$ is positive valued and satisfies

$$
c_{0} \Phi_{0} \leqslant \Phi \leqslant c_{1} \Phi_{0}+c_{2}
$$

for some positive constants $c_{1}, c_{2}$. As a consequence, by Gronwall's lemma (62) implies that

$$
\Phi(t) \leqslant M_{1} \Phi(0)+M_{2}
$$

where

$$
M_{1}=\exp \left(m / c_{0}\right), \quad M_{2}=\exp (C m), \quad m=\int_{0}^{\infty} \rho(t) \mathrm{d} t
$$

Since

$$
\|\varphi\| \leqslant|\Omega|^{1 / 4}\|\varphi\|_{L^{4}(\Omega)}, \quad\|u(t)\|_{L^{1}(\Omega)} \leqslant\|\theta\|_{L^{1}(\Omega)}+\left\|\theta_{\mathscr{H}}\right\|_{L^{1}(\Omega)}
$$

the boundedness of $\Phi_{0}(t)$ implies that of the energy norm $\mathscr{E}(t)$. This concludes the proof of the stability of the solution to (56)-(58) relative to the norm $\mathscr{E}(t)$.

\section{ACKNOWLEDGEMENTS}

The research leading to this paper was partially supported by Italian MIUR through the Research Project 'Mathematical models and methods in continuum physics'.

\section{REFERENCES}

1. Landau LD, Ginzburg VL. On the theory of superconductivity. In Collected Papers of L.D. Landau, ter Haar D (ed.). Pergamon: New York, 1965.

2. Fried E, Gurtin ME. Continuum theory of thermally induced phase transitions based on an order parameter. Physica D 1993; 68:326-343. 
3. Fried E, Gurtin ME. Dynamic solid-solid phase transitions with phase characterized by an order parameter. Physica D 1994; 72:287-308.

4. Frémond M. Non-smooth Thermodynamics. Springer: Berlin, 2002.

5. Bonfanti G, Frémond M, Luterotti F. Global solution to a nonlinear system for irreversible phase changes. Advances in Mathematical Sciences and Applications 2000; 10:1-24.

6. Fabrizio M, Giorgi C, Morro A. A thermodynamic approach to non-isothermal phase-field evolution in continuum physics. Physica D 2006; 214:144-156.

7. Brokate M, Sprekels J. Hysteresis and Phase Transitions. Springer: Berlin, 1996.

8. Kittel C. Introduction to Solid State Physics. Wiley: New York, 1966.

9. Penrose O, Fife PC. Thermodynamically consistent models of phase-field type for the kinetics of phase transitions. Physica D 1990; 43:44-62.

10. Fabrizio M. Ginzburg-Landau equations and first and second order phase transitions. International Journal of Engineering Science 2006; 44:529-539.

11. Berti V, Fabrizio M, Giorgi C. Well-posedness for solid-liquid phase transitions with a fourth-order nonlinearity. Physica D, in press.

12. Dryja M, Moszyński K. On Jeffreys model of heat conduction. Applied Mathematics 2001; 28:329-351.

13. Morro A, Ruggeri T. Non-equilibrium properties of solids obtained from second-sound measurements. Journal of Physics C: Solid State Physics 1988; 21:1743-1752.

14. Fabrizio M, Giorgi C, Morro A. A non-isothermal phase-field approach to the second-sound transition in solids. Nuovo Cimento B 2006; 121:383-399.

15. Coleman BD, Gurtin ME. Equipresence and constitutive equations for rigid heat conductors. Zeitschrift fur Angewandte Mathematik und Physik 1967; 18:199-208.

16. Fabrizio M, Giorgi C, Morro A. Free energies and dissipation properties for systems with memory. Archive for Rational Mechanics and Analysis 1994; 125:341-373.

17. Giorgi C, Marzocchi A, Pata V. Asymptotic behavior of a semilinear problem in heat conduction with memory. NODEA Nonlinear Differential Equations and Applications 1998; 5:333-354.

18. Fabrizio M, Lazzari B. On the existence and asymptotic stability of solutions for linear viscoelastic solids. Archive for Rational Mechanics and Analysis 1991; 116:139-152.

19. Amendola G, Carillo S. Thermal work and minimum free energy in a heat conductor with memory. Quarterly Journal of Mechanics and Applied Mathematics 2004; 57:429-446.

20. Golden M. Free energies in the frequency domain: the scalar case. Quarterly of Applied Mathematics 2000; 58: $127-150$.

21. Deseri L, Gentili G, Golden M. An explicit formula for the minimum free energy in linear viscoelasticity. Journal of Elasticity 1999; 54:141-185.

22. Fabrizio M, Morro A. Equivalent histories, minimal state and initial value problem in viscoelasticity. Mathematical Methods in the Applied Sciences 2005; 28:233-251.

23. Deseri L, Fabrizio M, Golden M. On the concept of a minimal state in viscoelasticity: new free energies and applications to PDEs. Archive for Rational Mechanics and Analysis 2006; 181:43-96.

24. Noll W. A new mathematical theory of simple materials. Archive for Rational Mechanics and Analysis 1972; 48: $1-50$.

25. Gilardi G, Rocca E. Convergence of phase field to phase relaxation models governed by an entropy equation with memory. Mathematical Methods in the Applied Sciences 2006; 29:2149-2179. 